\title{
No reliable evidence to guide initial arch wire choice for fixed appliance therapy
}

\author{
Abstracted from \\ Jian F, Lai W, Furness S, McIntyre GT, Millett DT, Hickman J, Wang Y. \\ Initial arch wires for tooth alignment during orthodontic treatment with fixed appliances. \\ Cochrane Database Syst Rev 2013; Issue 4. Art. No.: CD007859. DOI: 10.1002/14651858.CD007859.pub3. \\ Address for correspondence: Luisa Fernandez Mauleffinch, Review Group Co-ordinator, \\ Cochrane Oral Health Group, MANDEC, School of Dentistry, University of Manchester, \\ Higher Cambridge Street, Manchester, M15 6FH, UK. E-mail: luisa.fernandez@manchester.ac.uk
}

\section{Question: Which initial arch wire is most effective for fixed appliance therapy?}

Data sources The Cochrane Oral Health Group's Trials Register, the Cochrane Central Register of Controlled Trials (CENTRAL), Medline and Embase databases were searched. Conference proceedings and abstracts from the British Orthodontic Conference European Orthodontic Conference and the International Association for Dental Research were also searched together with the reference lists of identified studies. Study authors were contacted for additional information.

Study selection Randomised controlled trials of initial arch wire involving participants with upper and/or lower full arch fixed orthodontic appliances were included.

Data extraction and synthesis Study selection, data extraction and risk of bias assessment were carried out independently by at least two reviewers.

Results Nine RCTs with 571 participants were included in this review. All trials were at high risk of bias. All trials had at least one potentially confounding factor (such as bracket type, slot size, ligation method, extraction of teeth) which is likely to have influenced the outcome and was not controlled in the trial. None of the trials reported the important adverse outcome of root resorption. The comparisons were made between:

(1) Multistrand stainless steel initial arch wires compared to superelastic nickel titanium (NiTi) initial arch wires. There were four trials in this group, with different comparisons and outcomes reported at different times. No meta-analysis was possible. There is insufficient evidence from these trials to determine whether or not there is a difference in either rate of alignment or pain between stainless steel and NiTi initial arch wires.

(2) Conventional (stabilised) NiTi initial arch wires compared to superelastic NiTi initial arch wires. There were two trials in this group, one reporting the outcome of alignment over six months and the other reporting pain over one week. There is insufficient evidence from these trials to determine whether or not there is any difference between

This paper is based on a Cochrane Review published in the Cochrane Library 2013, issue 4 (see www.thecochranelibrary.com for information). Cochrane Reviews are regularly updated as new evidence emerges and in response to feedback, and the Cochrane Library should be consulted for the most recent version of the review. conventional (stabilised) and superelastic NiTi initial arch wires with regard to either alignment or pain.

(3) Single-strand superelastic NiTi initial arch wires compared to other $\mathrm{NiTi}$ (coaxial, copper $\mathrm{NiTi}$ (CUNiTi) or thermoelastic) initial arch wires. The three trials in this comparison each compared a different product against single-strand superelastic NiTi. There is very weak unreliable evidence, based on one very small study ( $\mathrm{n}=$ 24) at high risk of bias, that coaxial superelastic NiTi may produce greater tooth movement over 12 weeks, but no information on associated pain or root resorption. This result should be interpreted with caution until further research evidence is available. There is insufficient evidence to determine whether or not there is a difference between either thermoelastic or CuNiTi and superelastic NiTi initial arch wires.

Conclusions There is no reliable evidence from the trials included in this review that any specific initial arch wire material is better or worse than another with regard to speed of alignment or pain. There is no evidence at all about the effect of initial arch wire materials on the important adverse effect of root resorption. Further welldesigned and conducted, adequately-powered RCTs are required to determine whether the performance of initial arch wire materials as demonstrated in the laboratory, makes a clinically important difference to the alignment of teeth in the initial stage of orthodontic treatment in patients.

\section{Commentary}

As expected from a properly guided Cochrane Review this review follows generally accepted guidelines to conduct and report a systematic review. It has to be noted that this is an update from a review first published in 2010. An update after three years is well received, even though the conclusions remained unchanged. The stated objectives are clinically important when properly framed and the considered outcomes are important for both the clinician (level and alignment, root resorption) and the patient (pain intensity). One relatively significant deficiency of this review is not having included electronic databases that include articles published from Brazil, China and Turkey in their original languages. These countries have consistently published clinical trials in the last decade. Maybe some additional RCTs could have been missed.

Regarding the quality of included studies, only RCTs with a fully bonded dental arch were included. Although the number of included RCTs is respectable, the total number of included 
participants is not overall impressive. For the specific review questions the unit of analysis is the participant not the number of included teeth. When the methodological quality of the included RCTs is considered it becomes clear that the results need to be considered very cautiously due to the high risk of bias among all the studies.

None of the included studies did quantify or qualify the amount of root resoption produced. So there is no answer to that question. In regards to any specific type of wire marketed as an initial orthodontic wire being superior for level and alignment, this systematic review failed to find any consistent evidence. The same applies for pain intensity.

In summary, the available evidence failed to justify consistently the selection of any specific initial level and alignment orthodontic wire as a superior option. We have to keep in mind that lack of evidence is not automatically proof of no difference.

\section{Practice points}

- Based on this review results other factors should be considered by clinicians when selecting initial archwires. Factors such as cost, potential patients' allergy to specific alloys and initial amount of crowding in the three planes of space should be considered

- One major limitation that we will face in our quest to answer these questions is the large number of commercially available archwires at our disposal. Combination of factors such as archwire dimension, shape and composition will make it likely impossible to analyse all the potential combinations in a meaningful and lowrisk-of-bias fashion unless we increase the participation of private practices in research networks.

\section{Carlos Flores-Mir}

Division of Orthodontics, Department of Dentistry, Faculty of Medicine and Dentistry, University of Alberta, Edmonton, Canada Evidence-Based Dentistry (2013) 14, 114-115. doi:10.1038/sj.ebd.6400970 